\title{
Direct Measurement of the Formation Length of Photons
}

\author{
Kristoffer K. Andersen, ${ }^{1, *}$ Søren L. Andersen, ${ }^{1}$ Jakob Esberg, ${ }^{1}$ Helge Knudsen,,${ }^{1}$ Rune Mikkelsen, ${ }^{1}$ Ulrik I. Uggerhøj,
} Pietro Sona, ${ }^{2}$ Alessio Mangiarotti, ${ }^{3}$ Tjeerd J. Ketel, ${ }^{4}$ and Sergio Ballestrero ${ }^{5}$

(CERN NA63)

\author{
${ }^{1}$ Department of Physics and Astronomy, Aarhus University, DK-8000 Aarhus C, Denmark \\ ${ }^{2}$ University of Florence, Florence, Italy \\ ${ }^{3}$ Universidade de Coimbra, Portugal \\ ${ }^{4}$ VU University, Amsterdam, The Netherlands \\ ${ }^{5}$ University of Johannesburg, Johannesburg, South Africa \\ (Received 2 December 2011; published 16 February 2012)
}

\begin{abstract}
We report the first observation of a shoulder in the radiation spectrum from $\mathrm{GeV}$ electrons in a structured target consisting of two thin and closely spaced foils. The position of the shoulder depends on the target spacing and is directly connected to the finite formation length of a low-energy photon emitted by an ultrarelativistic electron. With the present setup it is possible to control the separation of the foils on a $\mu \mathrm{m}$ scale and hence measure interference effects caused by the macroscopic dimensions of the formation length. Several theoretical groups have predicted this effect using different methods. Our observations have a preference for the modified theory by Blankenbecler but disagree with the results of Baier and Katkov.
\end{abstract}

DOI: 10.1103/PhysRevLett.108.071802

PACS numbers: 13.40.-f, 12.20.Fv, 41.60. $-\mathrm{m}$

The well-known Landau-Pomeranchuk-Migdal (LPM) $[1,2]$ effect suppresses the low-energy part of the BetheHeitler (BH) [3] bremsstrahlung spectrum from an ultrarelativistic electron passing an amorphous material. The suppression is caused by multiple Coulomb scattering (MCS) within the formation length. For a photon with energy $\hbar \omega$ emitted by an electron with energy $E$ the formation length is [4]

$$
l_{f}=\frac{2 E(E-\hbar \omega)}{m^{2} c^{3} \omega},
$$

where $m$ is the electron mass, $c$ the speed of light, and $\hbar$ Planck's constant divided by $2 \pi$.

The LPM effect is mainly important for high-energy electrons emitting low-energy photons since the formation length is longest in that case. A simple estimate of when MCS within the formation length is important can be given by comparing the root mean square MCS angle over the formation length $\theta_{\mathrm{MCS}}$ to the typical photon emission angle [5] $\theta_{\gamma}=1 / \gamma \equiv m c^{2} / E$. When $\theta_{\mathrm{MCS}}>\theta_{\gamma}$ suppression is significant. This happens for photons with $\hbar \omega<\hbar \omega_{\text {LPM }}=$ $E^{2} /\left(E+E_{\mathrm{LPM}}\right)[4]$, where $E_{\mathrm{LPM}}=7.7 \mathrm{TeV} / \mathrm{cm} \times X_{0}$ and $X_{0}$ is the radiation length of the material. When the LPM effect is present, the almost constant $\mathrm{BH}$ radiation intensity, $\hbar \omega \frac{d N}{d \hbar \omega}$, is changed to a $\sqrt{\hbar \omega}$ behavior.

For a thin target the suppression effect is modified for photon energies low enough for the formation length to extend out of the target[6,7]. This so-called TernovskiiShul'ga-Fomin (TSF) effect has been investigated in recent years [8]. It was found that for a fixed photon energy, the decrease of the radiation intensity from the $\mathrm{BH}$ level to the LPM level for increasing target thickness, $\Delta t$, could be described by a simple logarithmic function of $\Delta t$. A simple estimate of the onset energy of this effect can be found by setting the formation length equal to the target thickness and isolating the photon energy. This is given by

$$
\hbar \omega_{\mathrm{TSF}}=\frac{E}{1+\frac{m c \Delta t}{2 \hbar \gamma}} .
$$

In the TSF regime the LPM suppression is lifted and the radiation intensity below $\hbar \omega_{\text {TSF }}$ is again almost independent of the photon energy. However, the intensity is reduced in magnitude compared to the $\mathrm{BH}$ level.

The successful measurements by the SLAC E-146 [9-11] collaboration of the LPM effect initiated considerable activity on the theory side. This also led to the investigation of a structured target consisting of two thin foils separated by a small gap. Three independent theoretical groups found a maximum in the calculated radiation spectrum from such a structured target [12-15]. The position of the maximum is directly connected to the formation length of photons at that energy. Since the formation length of a $1 \mathrm{GeV}$ photon emitted by a $200 \mathrm{GeV}$ electron is $60 \mu \mathrm{m}$, one can basically measure interference effects caused by the finite formation length with a micrometer screw.

An alternative way of understanding the appearance of the maximum in the radiation spectrum from a structured target is the following: consider first a target of a certain thickness where the LPM effect is important. If the target is cut into three pieces and the central part removed one also 
removes part of the suppression caused by MCS in this part of the target. High-energy photons created in the first target are in the $\mathrm{BH}$ regime. At intermediate photon energies LPM suppression occurs and at even lower energies the formation length of the photons extends into the gap and follow the TSF regime. For photons with a formation length that extends into the second target, LPM suppression is again present. Hence, this effect can in a simple picture be considered a suppression-alleviationsuppression effect caused by MCS. The position of the maximum in the radiation spectrum can simply be approximated by setting $\Delta t$ equal to the gap length $l_{g}$ in Eq. (2). In the case of foils of finite thickness one should use the distance between the foil centers. The alleviation effect that creates the shoulder is relatively small compared to the suppression caused by the second foil. Hence, the larger feature in the radiation spectrum from a structured target compared to a single foil is the extra suppression at lower energies caused by the second foil.

Calculated radiation spectra for different scenarios that are relevant for the present experiment are shown in Fig. 1. The Bethe-Heitler spectrum assumes that the interactions are independent. Hence it is only valid for thin targets and/ or incoherent interactions. The LPM suppression is shown for a semi-infinite target where boundary effects are

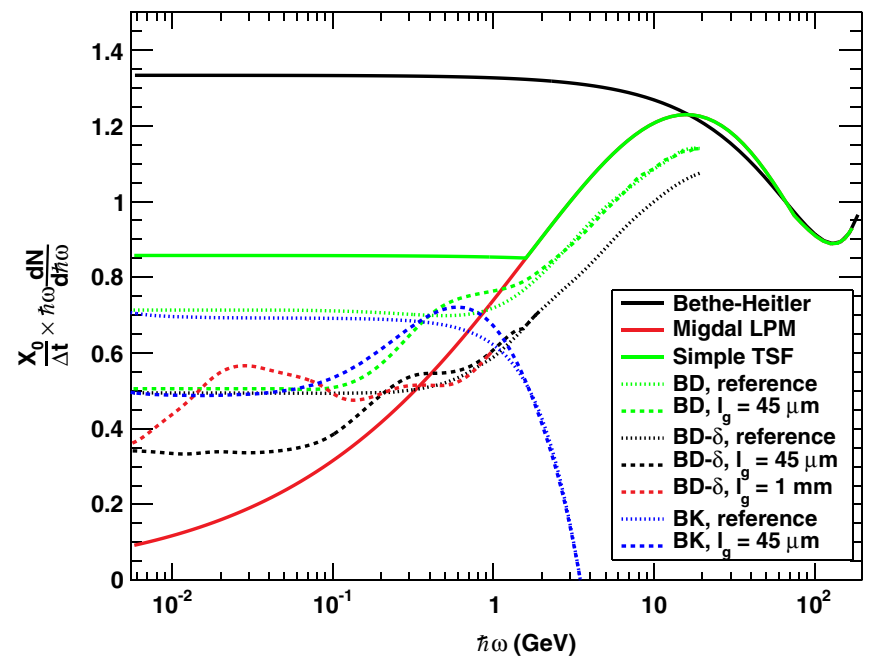

FIG. 1 (color online). Radiation intensity for a $197 \mathrm{GeV}$ electron passing a gold target. The spectra have been normalized to the radiation length and the target thickness so that the BetheHeitler level is 4/3. The Bethe-Heitler intensity is shown (black) together with the LPM effect (red), a simple model of the TSF effect (green) for a $26 \mu \mathrm{m} \mathrm{Au}$ target, a calculation using the formalism of Blankenbecler and Drell (BD) for a $26 \mu \mathrm{m} \mathrm{Au}$ target (green, dotted), a similar calculation using the theory of Baier and Katkov (BK) (blue, dotted) and for a sandwich target consisting of two $26 \mu \mathrm{m}$ foils separated by $45 \mu \mathrm{m}$ using BD (green, dashed) and BK (blue, dashed). The corrected BD theories $(\mathrm{BD}-\delta)$ are shown in black for the reference (dotted) and structured target (dashed). A BD- $\delta$ calculation for a target spacing of $1 \mathrm{~mm}$ is also shown (red, dashed) (see text for more details). neglected. It has been calculated using the approximations given by Stanev and collaborators [16]. The simple model of the TSF effect is based on models by Shul'ga and Fomin and asymptotic expansions of their result [17]. Using the theory of Blankenbecler and Drell (BD) [12] the spectrum is calculated for a single $26 \mu \mathrm{m}$ target (reference) and a structured target with a gap of $45 \mu \mathrm{m}$. Similar results have been found using the theory of Baier and Katkov (BK) [15] for the same situations. This theory is only valid for formation lengths much longer than the thickness of the individual target foils. The threshold energy $\omega_{\text {th }}$ given by Eq. (3.3) in [15] is for these experimental conditions $\omega_{\text {th }}=$ $1.01 \mathrm{GeV}$. The falloff around $1 \mathrm{GeV}$ is connected with this limitation and is also present in calculations performed by Baier and Katkov ([15], Fig. 3.1). The BD and BK theories generally agree (within 10\% [15]) and are close to calculations by Zakharov [14].

The BD theory was later corrected by Blankenbecler [13]. He used functional integrals for the averaging involved in the treatment of multiple scattering (this theory is denoted by $\mathrm{BD}-\delta$ ). The result is a quite substantial correction to the BD theory that lowers the energy of the shoulder in the spectrum of a structured target. It has not been possible to calculate the BD- $\delta$ theory for $l_{g}=1 \mathrm{~mm}$ above $1 \mathrm{GeV}$ to a satisfactory precision due to a heavily oscillating integrand. For $l_{g}=45 \mu \mathrm{m}$ the calculation has been performed up to $2 \mathrm{GeV}$. For both gap distances the calculations agree well with the reference curve at high photon energies as it should.

The NA63 experiment at CERN has investigated radiation emission from $197 \mathrm{GeV}$ electrons traversing a structured target consisting of two $26 \mu \mathrm{m}$ thick $\mathrm{Au}$ foils separated by distances of $45 \mu \mathrm{m}, 1$ and $10 \mathrm{~mm}$. The setup is shown in Fig. 2. A tertiary beam of $197 \mathrm{GeV}$ electrons from the CERN SPS is used at the H4 beam line in the North Area. The normalization trigger is defined by two $0.5 \mathrm{~mm}$ thick scintillator counters and a $\varnothing 9 \mathrm{~mm}$ hole scintillator. After passing the target assembly the initial electrons are deflected by a $4 \mathrm{~m}$ long $0.157 \mathrm{~T}$ field. This deflects the initial beam outside the $\varnothing 7.5 \mathrm{~mm}$ BGO detector at a distance of $65 \mathrm{~m}$ from the magnet and into the lead glass calorimeter (LG). It is crucial for the experiment to use a low magnetic field since synchrotron radiation is the dominant background at low energies. With the used magnetic field the critical energy of the synchrotron radiation is $8 \mathrm{MeV}$. The BGO detector is used to measure the $\mathrm{MeV}$

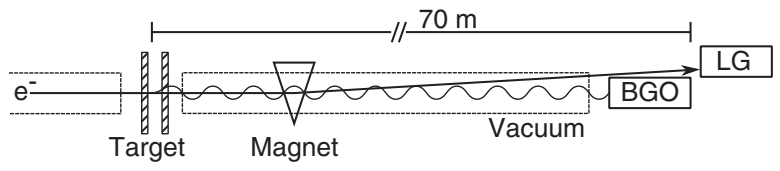

FIG. 2. Experimental setup (not to scale). A $197 \mathrm{GeV}$ electron beam passes the target assembly and is deflected by a magnet setup. Approximately $65 \mathrm{~m}$ downstream a BGO detector is placed in the photon beam and the deflected electron beam is measured by a lead glass calorimeter (LG). 
photons created in the target. It has previously been described [8] and in the present experiment we verified and used the same settings and calibration as in previous experiments. The uncertainty is expected to be less than $\pm 10 \mathrm{MeV}$. Furthermore, we split the BGO signal so that two different amplifications could be used, called BGO and a higher amplification called BGOA. The LG detector is used for cuts in the electron spectrum. Since we focus on photons with $\hbar \omega \ll E$ interesting events will have a highenergy electron accompanying them. Furthermore, a veto scintillator is placed in front of the BGO to discard events where the photon has pair converted in flight. With these settings we are able to measure photon energies from around $3 \mathrm{GeV}$ and down to below $100 \mathrm{MeV}$ eventually being limited by the onset of (pileup) synchrotron radiation around $50 \mathrm{MeV}$.

A major challenge for the $\mathrm{BGO}$ detector is high-energy photons which are an inevitable background. The shower produced in the BGO crystal by a high-energy photon overloads the PMT and amplifier and leaves the detector inefficient for up to more than $0.5 \mathrm{~ms}$. To be able to remove these events from the data set we have marked all events following a high-energy photon if they happen within 50, 200 , or $500 \mu \mathrm{s}$ after the critical event. Furthermore, we have also measured the value of the detector baseline just before an event. If the baseline is shifted the amplifier does not work as expected and the event is rejected. Approximately $25 \%$ of the events have a significant baseline offset, $30 \%$ are within $500 \mu$ s of a high-energy photon event and $35 \%$ are affected by either of the two. Since two different amplifiers have been used, one can generally not expect the baseline to be reached at the same time. The baseline cut used for the BGOA data is more restrictive than the cut used for BGO data and hence after applying the baseline cut there is $\sim 33 \%$ more events in the BGO data set compared to the BGOA data set. This difference lead to fluctuations between the BGO and BGOA data sets, which reflects the systematical uncertainty related to the choice of baseline cut. In the analysis we have used the $500 \mu$ s exclusion and individual baseline cuts if nothing else is stated.

The thicknesses of the two Au foils have been measured to be $26 \pm 1 \mu \mathrm{m}$ and $27 \pm 1 \mu \mathrm{m}$ corresponding to a total of $1.6 \%$ radiation length. When the foil separation is large $(\sim 10 \mathrm{~mm})$, the radiation spectrum is expected to correspond to two independent TSF spectra. This is our reference measurement. To ensure that the foils are parallel, one of the foils is placed in a spring-loaded gyro. When the translatable foil is moved towards the other foil and pressed against it, the two foils will align.

The gap length is determined by three different methods. Initially, the position of the translatable foil is controlled in micrometer steps and read out. It can also be directly measured with a Mahr MarCator placed on the translatable stage with a resolution of $1 \mu \mathrm{m}$. Finally, the capacitance between the two foils is measured. For small distances we assume that the capacitance is given by the sum of a parallel-plate capacitor and a capacitance offset. The distances measured with these three techniques agree. Hence, we are confident that the gap length is determined within the precision of $\sim 5 \mu \mathrm{m}$. The surface variations of the foils when mounted has been investigated by laser reflection, and it was found to be less than $\pm 2 \mu \mathrm{m}$.

We have made Monte Carlo simulations to verify the measured radiation spectra. The MC includes a BetheHeitler background, synchrotron radiation from the bending magnet and radiation from the structured target. This has been calculated using either the $\mathrm{BD}$ or the $\mathrm{BD}-\delta$ theory. We assume that the detector efficiency can be described by a simple 2 nd order polynomial and find this by fitting the measured background radiation to MC simulations. The efficiency is in good agreement with earlier experiments [8].

In Fig. 3 BGO data is shown together with MC simulations based on the BD- $\delta$ theory. The agreement is satisfactory which shows the reliability of the data and supports our interpretation of them.

The ratio between the $l_{g}=45 \mu \mathrm{m}$ spectrum and the reference spectrum (for both spectra the background has been subtracted) is plotted in Fig. 4. In this ratio, the influence of the detector efficiency is eliminated. Together with the data two Monte Carlo simulations based on the $\mathrm{BD}$ and $\mathrm{BD}-\delta$ theories are plotted, as well as a number of theoretical curves. The BGOA (black stars) and the BGO (red circles) signals are plotted at the lowest energies and above $80 \mathrm{MeV}$, respectively. In the overlap region from 80 to $800 \mathrm{MeV}$ these two measurements agree as expected. The MC follows closely the theoretical curve of the BD- $\delta$ above $300 \mathrm{MeV}$. At lower energies pileup causes the MC to fall below the theoretical curves, but the position of the peak is almost unaffected by this. The BD and BK theories both lie substantially higher with a peak position around $900 \mathrm{MeV}$ and in clear disagreement with the data, as is also confirmed by the $\chi^{2}$ values. These are calculated in the interval from $20 \mathrm{MeV}$ to $2 \mathrm{GeV}$ with

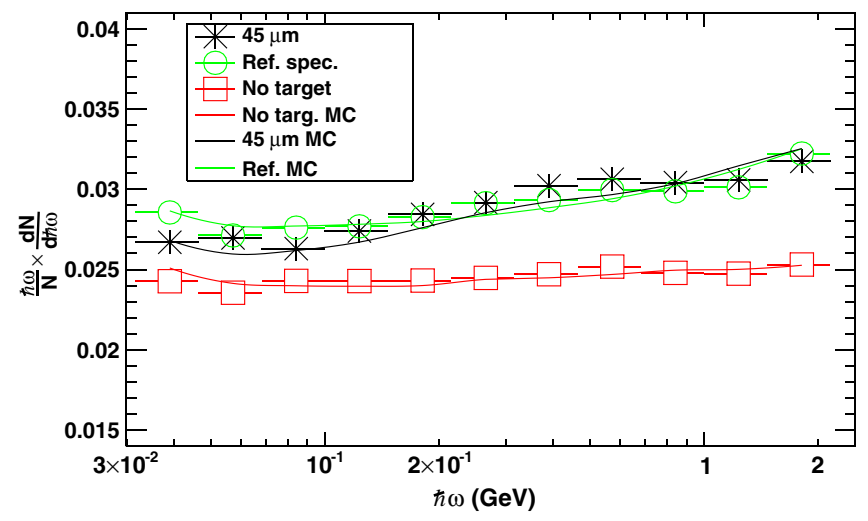

FIG. 3 (color online). BGO data and MC simulations. No target data are shown with red squares, reference data with green circles and $45 \mu \mathrm{m}$ separation with black stars. MC is shown with lines. No target in red, reference in green, and $l_{g}=45 \mu \mathrm{m}$ in black. The horizontal bar is the bin width. 


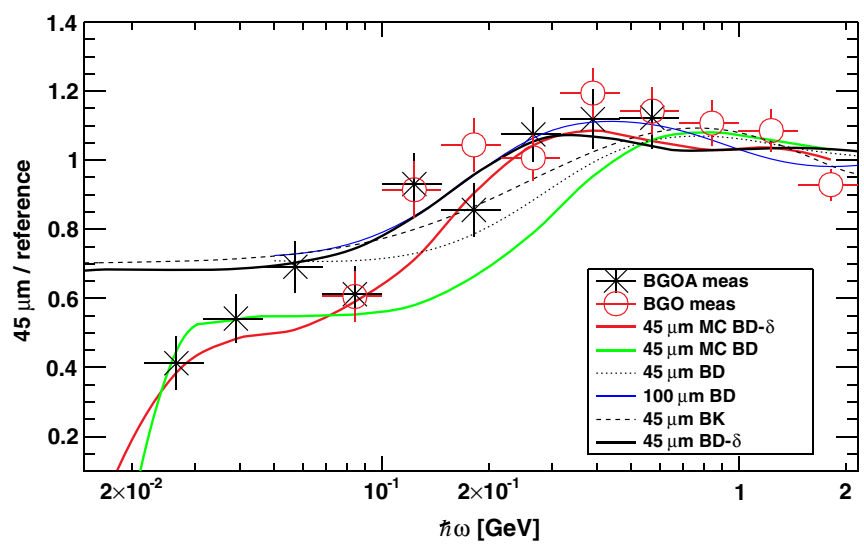

FIG. 4 (color online). The ratio between a measurement with $l_{g}=45 \mu \mathrm{m}$ and the reference measurement. Both spectra have been background subtracted. The BGOA measurements are shown with black stars and BGO with red circles. Monte Carlo simulations using $\mathrm{BD}$ (green) and $\mathrm{BD}-\delta$ (red) theories are also shown. Several theoretical curves based on BD and BK theory are shown for different gap sizes. The horizontal bar is the bin width and the vertical bar is the statistical errors.

BGOA data used below $0.1 \mathrm{GeV}$ and BGO data above. The $\chi^{2} /$ d.o.f. $\quad$ values are $\chi^{2} /$ d.o.f. $=25.0 / 12=2.09$, $\chi^{2} /$ d.o.f. $=75.4 / 12=6.28$ and $\chi^{2} /$ d.o.f. $=205 / 12=$ 17.1 for the BD- $\delta$ theory, BD theory and constant one (no effect), respectively. Hence, the $\chi^{2}$ probability ( $p$-value) is $\sim 1.5 \%$ for the BD- $\delta$ theory and negligible for the other two. Since both the BK theory and the theory of Zakharov give results that are close to the BD theory these disagree with our $l_{g}=45 \mu \mathrm{m}$ measurement.

A similar measurement with a $1 \mathrm{~mm}$ spacing has been made. With this spacing the resonance is expected to be around $60 \mathrm{MeV}$. In Fig. 5 the ratio between the $1 \mathrm{~mm}$ spectrum and the reference spectrum is plotted. Because of pileup of synchrotron radiation the effect is somewhat washed out and we do not observe any clear signal. Both the BD and the BD- $\delta$ theories are plotted. For this separation a simple $\chi^{2}$ analysis shows that the data are closer to 1 than to both the BD and BD- $\delta$ theories.

In conclusion, we reported here the first measurements where a shoulder in the radiation spectrum from a structured target has been observed. The position of the shoulder is closely related to the formation length of photons and one can basically measure this on a micrometer scale with the present setup. Furthermore, we showed that for a target separation of $45 \mu \mathrm{m}$ our data have a preference for the corrected theory of Blankenbecler [13]. The uncorrected BD [12] theory gives noticeably different results. For the $1 \mathrm{~mm}$ separation the data show no clear effect.

In this experiment the suppression mechanism was caused by multiple coulomb scattering. Another possibility would be to replace the second foil with a strong laser field. In this case Compton scattering would affect the electrons and probably suppress the emission of radiation. The position of the laser field would determine what photon

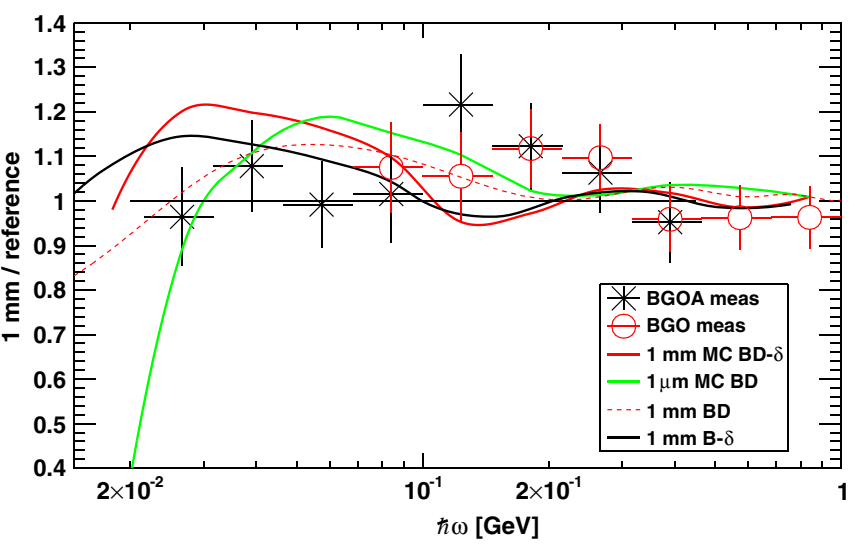

FIG. 5 (color online). The ratio between a measurement with $l_{g}=1 \mathrm{~mm}$ and a reference measurement. Both spectra have been background subtracted. The BGOA measurements are shown with black stars and BGO with red circles. Monte Carlo simulations using BD (green) and BD- $\delta$ (red) theories are also shown. The horizontal bar is the bin width and the vertical bar is the statistical errors.

energies are suppressed like the gap distance determines the shoulder in the radiation spectrum from a structured target. However, a very strong laser field is required to get a significant effect. Such laser fields have been used in experiments with multi-GeV electron beams where nonlinear QED effects was observed [18].

We wish to thank Per Christensen and Poul Aggerholm for technical support with the experimental setup and DAQ system.

*kka@phys.au.dk

[1] L. Landau and I. Pomeranchuk, Dokl. Akad. Nauk SSSR 92, 535 (1953); The Collected Papers of L. D. Landau (Pergamon, New York, 1965).

[2] A. B. Migdal, Phys. Rev. 103, 1811 (1956).

[3] H. Bethe and W. Heitler, Proc. R. Soc. A 146, 83 (1934).

[4] S. Klein, Rev. Mod. Phys. 71, 1501 (1999).

[5] J. Jackson, Classical Electrodynamics (Wiley, New York, 1999), 3rd ed.

[6] F. Ternovskii, Zh. Eksp. Teor. Fiz. 39, 171 (1960) [Sov. Phys. JETP 12, 123 (1960)].

[7] N.F. Shul'ga and S.P. Fomin, Sov. Phys. JETP 86, 32 (1998).

[8] H. D. Thomsen et al., Phys. Rev. D 81, 052003 (2010).

[9] P. L. Anthony et al., Phys. Rev. Lett. 75, 1949 (1995).

[10] P. L. Anthony et al., Phys. Rev. Lett. 76, 3550 (1996).

[11] P. L. Anthony et al., Phys. Rev. D 56, 1373 (1997).

[12] R. Blankenbecler, Phys. Rev. D 55, 190 (1997).

[13] R. Blankenbecler, Phys. Rev. D 55, 2441 (1997).

[14] B. G. Zakharov, Pis'ma Zh. Eksp. Teor. Fiz. 64, 737 (1996) [JETP Lett. 64, 781 (1996).]

[15] V. N. Baier and V. M. Katkov, Phys. Rev. D 60, 076001 (1999).

[16] T. Stanev et al., Phys. Rev. D 25, 1291 (1982).

[17] U. I. Uggerhøj et al., Phys. Rev. D 72, 112001 (2005).

[18] C. Bamber et al., Phys. Rev. D 60, 092004 (1999). 